\title{
Managing and Valuing Diversity in the South African Public Healthcare System
}

\section{Rubeshan Perumal \\ Sadhasivan Perumal}

\begin{abstract}
Human capital remains a critical component of healthcare, particularly in low and middle-income countries where there is a dire shortage of professionals. Recruiting and retaining health professionals has become a key strategic focus in South Africa. Innovative recruitment strategies coupled with increasing access to higher education and training has resulted in an increasingly diverse workforce. Identifying, appreciating, and managing this diversity across all diversity dimensions is important for both social and economic reasons. These efforts are likely to provide an opportunity for optimizing productivity while building a cohesive workforce. This paper explores the impetus and possible methods for effective diversity management in the South African public healthcare environment.
\end{abstract}

Keywords: Diversity, healthcare management, diversity management, transformation, human resources

\section{Introduction}

The South African healthcare system currently faces substantial systematic, operational and environmental challenges as it evolves under current resource constraints. Effectively managing the public healthcare sector, in particular, will require the application of sound principles of public administration and management, with serious considerations for strategic functions outside of the core business of clinical care. The most pervasive of such functions is arguably the strategic management of the critical human capital upon which the system 
relies, and without which failure at all levels would be inevitable. Healthcare, by its service nature, is a labour intensive operation (Siddiqui \& Kleiner 1998). The interface between healthcare workers and healthcare users serves as the point of exchange for the health commodity, and demonstrates the centrality of the healthcare workforce in ensuring the delivery of acceptable, efficient and valuable services. The intensive use of labour therefore, warrants focus on human resource practices and management. Perhaps the most radical change in the South African healthcare landscape over the past decade has been the diversification of the workforce, and the intermingling of these diverse human elements in microcosms of the new South Africa. The entry of previously nondominant, marginalized and disadvantaged groups adds depth to the challenge of diversity in the South African healthcare system(Human 2005). Acknowledging and responding to the challenge of diversity brings consequent gains, and conversely failing to do so will almost certainly carry economic, moral and legislative penalties (Cavaleros et al. 2002).

\section{The Concept of Diversity}

Diversity is rapidly becoming one of the most defining themes of our time. In its broadest sense diversity refers to differences in a multitude of attributes between individuals or groups. Uys (2003) describes three categories of diversity, namely functional diversity which refers to differences based on organizational functions and tasks, business diversity which refers to products and services offered and workforce diversity which implies different types of employees with different types of attributes. Conventional notions have included elements such as race, culture, gender and age, with the scope of diversity now having been widened to include spirituality, language, disability, physique, sexuality, educational level, skills, expertise, management styles, parental status, marital status, family background, vocational interests, career aspirations, geographic differences, social status, physical and mental conditions, communication styles, as well as personality attributes and working styles(Cavaleros et al. 2002; Greybe \& Uys 2001; Human 2005; Uys 2003; Narsee 2005). Political outlook and affiliation has become a significant force on the South African diversity landscape, affecting the composition and power distribution in many organizations (Nel et al. 2004).

Diversity can also be described as those human qualities which distinguish one individual or group from another. These qualities may further 
be categorized as being either unchangeable or changeable. Unchangeable or primary dimensions of diversity refer to differences that are inherent and fixed (Walt \& Du Toit 1997). These form the individual's self-image and his or her filters through which the world is seen and experienced. The assumption is that the greater the number of primary differences between people, the more difficult it is to establish trust and mutual respect. This leads to 'culture clashes' which could have devastating effects on human relations in the organization(Cilliers 2007). Changeable or secondary dimensions of diversity refer to differences which are modifiable or variable(Walt \& Du Toit 1997). These dimensions normally add complexity to the individual's self-image. The interaction between the primary and secondary dimensions shapes the individual's values, priorities and perceptions. The assumption is that effective human relations among diverse employees in the organization are possible only when the differences are accepted and valued (Cilliers 2007).

\section{The Imperatives for Understanding and Managing Diversity}

Diversity, as an organizational issue, has its provenance in the United States of America and Western Europe when the inclusion and equity of women and minority groups in the workforce was beginning to be addressed (Inyang 2007). With the increasing heterogeneity of the workforce, effectively managing diversity has become an imperative to achieving organizational competitiveness and ultimately success (Inyang 2007; Maxwell \& Rijamampianina 2002). Applying biological principles, failure to adapt to the changing composition of the workforce will result in a weaker organization which must ultimately succumb to the law of natural selection, where only the fittest, most competitive and responsive organizations survive.

In South Africa globalization, democratization, and Africanization have been significant forces which have shaped both the workforce and the customers it serves (Anca \& Vazquez 2007; Cilliers 2007). These three forces have not acted in isolation, but rather in concert. Democratization, post 1994, brought with it the challenges of globalization and increased interaction within the continent (Cilliers 2007). The re-arrangement of the South African health system post-apartheid has also meant that access to healthcare has been improved for the majority of South African who may not ordinarily have accessed organized healthcare institutions, thus changing the 'customer profile'. 
The imperatives for managing and valuing diversity come from both the external business case as well as the social-moral case. Gordon (1995) as cited in Cavaleros (2002) revealed that diversity contributes to an organization's competitive advantage, and improves its financial performance. However, unlike the private sector, the public sector cannot be limited by the pursuit of profitability, but should also reflect the state's commitment to equity, social justice and the protection of human rights (Uys 2003). A diverse workforce contains a vast amount of creativity and potential for innovation which may arise from the multitude of skills, abilities, thinking styles, and aptitudes of the employees. A diverse organization is able to capture new markets, maintain a broader social perspective, and exploit its multiculturalism by tapping into all market groups (Cavaleros et al. 2002). The ability of the organization to connect with its 'customers' is also enhanced by its multiculturalism and diversity.

Allopathic care has its roots firmly in the western paradigm of medicine, in which science if often viewed and practiced outside of the local social context. In societies such as South Africa, where traditional medicine remains a critical, underestimated and perhaps undervalued modality, there has been a growing recognition that maintaining an exclusive allopathic system fails the majority of healthcare users and insults the context in which the services are delivered. Any future endeavour to integrate indigenous traditional health practices into the existing system is certain to fail unless diversity is energetically addressed.

The National Health Act of 2003 declares the establishment of a district health system (RSA 2003). The organizational implications of this system include a flatter structure with a focus on decentralization which has been shown to increase reliance on participative group processes compared with hierarchical, highly centralized structures (Uys 2003). The centrality of the group in identifying and resolving organizational problems is not without its own set of disadvantages, which include intra-group conflict and coordination. Effectively managing the functional, cultural, ethnic, racial and gender differences within groups is essential to the success of the entire district health system.

One of the main reasons for addressing diversity arises from the belief that service users are better served by a diverse organization. Moreover, a socio-politically responsive organization is strategically positioned for understanding and delivering to its broad customer base, as well as attracting 
the support of customers who value the responsiveness of the organization (Human 2005). In South Africa, the political imperatives for actively pursing diversity in the workforce are intimately related to the proactive role that the government has taken in improving diversity in organizations (Human 2005). The health system, under the governance of the state, is expected to comply and further the agenda of the various pieces of legislation geared toward this aim. Notwithstanding the reorganization of the health system and recent focus on the decentralization of power, even the most geographically distant health services remain firmly within reach of the overarching policies and influence of the central authorities. Apart from binding legislation, the National Human Resource Plan for Health inherently acknowledges the challenge and opportunities of diversity in its strategic objectives (DOH 2006). Specifically, establishing shared values, balancing worker categories, aligning competencies, creating a culture of valuing all workers, optimization of relations with foreign professionals, and a national commitment to human resource planning are key objectives which demonstrate the government's responsiveness to workforce diversity (DOH 2006; Gill 1996; Greybe \& Uys 2001). Beyond the demand for organizations to be contextually relevant and socio-politically correct, at the strategic and operational level productivity remains a crucial goal. Maximizing the productivity of the workforce is central to the success of the organization and can only be achieved if employees are motivated, confident and satisfied. A diverse workforce is likely to enjoy the benefits of a non-prejudicial, multi-cultural work environment and may translate the experience into improved outputs. The valuing and fostering of diversity in the public healthcare sector may have significant implications for maintaining harmony in the workplace and improving the challenging retention rates within the service. Growing emphasis on the role of multidisciplinary teams in healthcare provide further justification for the role of diversity management in enhancing clinical care. Stereotypes within an organization, on the other hand, can prevent managers from seeing and valuing the potential in his or her subordinates. Unduly fixed beliefs about competencies and inherent abilities do not allow the potential of a diverse workforce to be fully exploited, but rather result in the underutilization and underestimation the organization's human resources.

The public health workforce is undeniably becoming increasingly demographically diverse. The de-racializing of health professions and the implementation of equity quotas, together with the broader social changes 
presents novel challenges to public service managers. The current spurt of male nurses, for example, necessitates the reconsideration of the previous genderexclusive professional nomenclature. The movement of historically geographically contained ethnic groups beyond the boundaries of the past creates complexities even within the construct of race. Lessons learnt from the more liberal west, socio-political interest groups, and the direction of national legislation tell us that the workforce of the future is likely to include people with more diverse sexual orientations, religious beliefs, spiritual perspectives, physical abilities and mental abilities (Uys 2003). Health institutions in the public service are expected to lead the way in non-discrimination on the basis of health status, sexual orientation, physical abilities and mental abilities. This must be achieved by diversifying the health workforce itself (internal commitment) as well as promoting the rights and interests of non-dominant groups in a broader social discourse (external commitment). In no other sector of the public service is the influx of foreign professionals more relevant. International and continental agreements to cope with severe skills shortages have become a striking feature of the rural health service. The National Health Act of 2003 acknowledges this by containing the principles underpinning the recruitment of foreign professionals, as well as provisioning the inclusion of foreign professionals into the national skills mix. The current efforts to revitalize the health professions and compensate for the severe skills shortages faced by the system have led to a large contingent of young employees. In addition, pressures to transform the workforce have also meant that younger workers of designated groups, for example, may come to lead a team of individuals who may be older than them. The impact of this age dynamic within the organization must be anticipated.

Careful evaluation of international and local failed attempts to dull the impact of diversity in organizations by propagating the view that professional or workplace similarities between individuals and groups outweigh private differences between them, demonstrates that the 'melting-pot' approach is severely deficient (Uys 2003). While a pervasive and inclusive organizational culture aids in creating bonds between employees, it does not detract from the quintessential realities of diversity. It may be argued that celebrating and sharing differences is a human need that must be satisfied in order for all parties to be maximally productive.

A critical analysis of diversity in the workforce is incomplete without a realization that diversity has disadvantages too. The pursuit of unity in 
diverse organizations can sometimes lead to convergence, where employees are encouraged to embrace their similarities and to act and think in similar ways (Schwella et al. 1996). Communication and integration of actions become more difficult in situations of diversity. In the context of service delivery in the health system, clinical care can be significantly compromised by diversification of the workforce, when the 'customer profile' of the public health system has lagged behind in the process of diversification. While representation in the workforce is aimed at mirroring and creating a microcosm of society at large, the socio-economic history of the country dictates that representation of public health service users does not accurately reflect the demographics of the South African population. Over-representation of the poor and historically marginalized is an overt manifestation. Language, cultural and ethnic barriers between the service provider (doctor) and the service user (patient) introduce challenges to the clinical assessment and management of the service user, and may lead to frustration of the competent but sociallydisconnected service provider.

\section{Managing and Valuing Diversity}

Diversity Management is the acceptance of the diverse nature of individuals and groups within society, and indeed within our organizations. It recognizes the visible and non-visible differences which need to be harnessed in order to create a work environment that is conducive to maximizing productivity and exploiting the full potential of each individual without placing any one person or group at an advantage or disadvantage (Inyang 2007). In retrospect, diversity management originally sought to address demographic inequalities at the organizational level with much attention having been paid to the establishing of equitable race and gender quotas. This discrimination and inequity paradigm directed diversity management activities toward the goal of 'representation' (Greybe \& Uys 2001). This superficial view of diversity was deficient in approach to understanding and levering the complexities of differences. Greybe and Uys (2001) describe a shift in the paradigm in the 1990 's to one of admission and legitimacy, which followed the realization that the needs of a diverse customer base are best served by a diverse organization that values its own diversity. The strong management focus on augmenting the service or product and customer satisfaction eclipsed the goal of grappling with the social issues of diversity, and the intricacies of workforce diversity. More 
recently diversity has been seen as an opportunity to incorporate the diverse perspectives of employees on strategic markets, products, services, practices and cultures (Greybe \& Uys 2001). Together with this new paradigm of learning and effectiveness, comes the insight into the true value that critical engagement with the challenge of diversity can bring to an organization. Quite clearly, the imperatives for managing diversity has stemmed from the propagation of the 'business case' and the promise of economic gains. Valuing diversity, on the other hand, have evolved around moral and ethical imperatives to recognize and appreciate culturally diverse people (Uys 2003). Programmes designed to 'value diversity' have made great strides in addressing diversity at the individual and inter-personal levels, but usually do not ascend to the organizational level. Consequently, organizational issues and organizational systems are excluded from the process of redress (Uys 2003). While valuing diversity aims to recognize and tolerate, managing diversity seeks to harness and capitalize on sensitivity to salient differences to create a more dynamic and competitive organizational culture (Uys 2003).

\section{Managing Diversity and Affirmative Action}

A further distinction that is important to make is that between managing diversity and affirmative action. Affirmative action falls under the broad umbrella of equal opportunity, which is legislated through the Employment Equity Act of 1998. The key principle underlying employment equity includes the equal treatment of all individuals regardless of the race, gender or ethnic origin. Perumal (1994) suggests that Affirmative Action is an adjunct to employment equity aimed at the elimination of discrimination against those persons previously treated unfairly by the employment practices and policies of a given institution, and rightly recognizes its role a tool for social justice in so far as it entails a systemic programme for bringing social opportunities and material benefits to a target group who would otherwise be deprived. While Affirmative Actions seeks to eliminate the barriers, racism and prejudice faced by designated groups in obtaining employment, managing diversity takes the more holistic approach of recognizing the unique dimensions of each individual and endeavors to harness the full productive and personal potential of the employee (Inyang 2007; Perumal 1994; Uys 2003). Interesting, affirmative action has contributed to the growing diversity of the public service and serves as the legislative impetus for pursing diversity programmes. In the 
main, however, affirmative action remains an institutional obligation under political, social and legal compulsion while the impetus for managing diversity is primarily an economic driver supported by the business case. Affirmative action programmes are directed at changes at the group level and are strategically managed by the human resource manager, while diversity management has its influence on the individual level and is controlled through the process of continuous feedback by the individuals themselves, with the involvement of all managers. The pluralistic, proactive, inclusive, and longterm nature of diversity management contrasts with the group-based, reactive, socially exclusive, relatively short term nature of Affirmative Action (Human 2005; Hunt 2007; Uys 2003). Pursuing affirmative action alone, in the hope that the representation it brings about will contribute to the management of diversity in the organization is an ill-founded assumption that threatens to be more crippling to the organization than enabling. Optimizing the potential of the workforce is essentially a managerial task, best achieved through managing its inherent diversity.

Currently affirmative action measures are being implemented in the selection, training and hiring of healthcare workers. Medical schools, in particular, have actively pursued racial quotas in their enrolment strategies as a means to effecting change in the medical profession. Notwithstanding this, the severe skills shortages in the health sector have prevented the maximal implementation of affirmative action policies, as suitable affirmable candidates are not readily available, and existing job, resource, and time-frame constraints prevent the selection and development of candidates who show 'potential' to perform. Diversity management, too, faces considerable challenges in the public service. The absence of benchmarks in the public service is a major limitation to managing diversity. In addition, the severe capacity and resource constraints in the health sector places diversity management low on the priority list of many managers who do not recognize it as a strategic and urgent need. Hospitals are large bureaucratic organizations, and change will, predictably, come slowly (Gay \& Bamford 2007). The current political climate of the country suggests that a turn toward elements a developmental state with increased involvement of the state in sectors such as health and education, potentially increasing existing bureaucracies. The lack of accountability for diversity management within the public service enables the perpetuation of the status quo. Arguably the greatest challenge facing long term, sustainable diversity management is the inequalities in education and training of future 
healthcare professionals. Current disparities in opportunities for education translate into differential access to employment (Inyang 2007). The healthcare sector is directly affected by the failure of the South African education schooling system to produce students who are adequately skilled in mathematics and science, and who are eligible for entry into institutions of higher learning. The inherent gender and racial disparities in primary and secondary education cannot be meaningfully corrected at the tertiary education level, and will inevitably manifest in the workforce. Preparedness of public health service managers and employees is lacking and will need to be addressed through awareness programmes and training. Diversity management would certainly not have been included in the conventional management skills set of many present managers in the system. In addition, many managers in the public health service, particularly hospital managers, have little or no general management education, let alone formal training in diversity management. Closing the gap between overt, on the ground needs of the health services and softer elements such as diversity management will be crucial to the efficient functioning of the system. Penceliah (2003) describes the recent interest in the role of emotional intelligence in public service managers. The ability to perceive and be aware of emotions, and to use this knowledge to discern the feelings underlying interpersonal communication is critical when dealing with the sensitivities of diversity management (Mullins 2005; Penceliah 2003).

\section{Strategies for Managing Diversity}

The multi-faceted, complex nature of diversity makes crafting and executing effective strategies for diversity management a challenging task. Understandably, there have been several models and strategies put forward by a multitude of stakeholders. Greybe and Uys (2001) suggest the following consolidated, comprehensive and pragmatic approach, which has been adapted for application in the South African public healthcare sector:

\section{Create Diverse Organizations}

Diverse organizations are more likely to recognize the need for diversity management and provide the heterogeneity that is required to critically engage with the challenge of diversity, while identifying the opportunities that arise from effective management. An organizational culture that values the diverse 
perspectives and contributions of all its employees is crucial to the successful management of workforce diversity.

\section{Create a Mission and Organizational Vision}

A shared organizational mission is essential to consolidate the diverse interests and perspectives of the heterogeneous workforce. It serves as both a bonding and a directive strategy within the organization. The mission and vision must be realized through strategic and operational plans that set targets and provide for monitoring of the diversity management activities.

\section{Introduce Creative Leadership}

Dynamic and visionary leadership is necessary as the driving force for diversity management in the organization. A radical approach is not necessary, whereas stability and balance amongst the diverse employees is crucial. A participatory leadership with 360-degree feedback is useful, but comes with the responsibility of having to provide the personnel with sufficient knowledge and information on the process. The fostering of an emotionally intelligent leadership will have positive outcomes on the management of diversity within the organization.

\section{Involve Senior Management Actively}

Senior management should be actively involved in pursuing diversity management within and outside of the organization. Attendance at cultural festivals and other cultural settings promotes the image of the organization and reflects its commitment to diversity management. Forming alliances with prominent cultural organizations in the community may also be a meaningful exercise for senior management, and sets the example for becoming knowledgeable about the different cultures and ethnicities in the community. A culture conscious management team is open to consultation and is able to establish and communicate diversity priorities to all stakeholders. Significantly, the commitment of senior management is essential in order for diversity management programmes to receive the kind of resource support that is necessary for its effectiveness. The potential conflict between short-term concentration on targets and the long term management of diversity must be prevented. 


\section{Ensure Effective Interpersonal and Inter-Group Communication}

The success or failure of an organization's diversity management strategies will depend largely on effective, clear and unambiguous communication. Constant updates and feedback must be encouraged through the use of newsletters, diversity training, workshops, staff attitude surveys and regular evaluation. Communication, in itself, has the potential to act as a tool for change and exchange. Ideas, knowledge and culture can all be expressed through interpersonal and inter-group communication. Diverse organizations, by the multi-cultural nature, however face inherent risks of miscommunication and difficulties in maintaining effective communication. Meanings and connotations are subliminally added to all communication as it is filtered through our social and cultural experience. Dialogue and repeated transmission of important information may help in reducing miscommunication. With 11 official languages, the South African public health service will have to take account of the multi-lingual, multi-ethnic nature of both its workforce and service users. Notices, health education, and official documents may require translation into the locally relevant language.

\section{Create Opportunities for Positive Diverse Interaction}

Historical racial segregation has been the hallmark of the past South African social and political landscape. The remnants of this divisive history remain with us and fuels misunderstandings. Positive social interaction in the form of sport, cultural festivals, workplaces and inter-religious gatherings can be used to market diverse communities.

\section{Develop Partnerships}

The public health service operates in a dynamic environment with numerous stakeholders and potential partners. Linkages with the private sector, pharmaceuticals, business, communities, religious organizations, professional associations, non-governmental organizations and inter-sectorial partners can be used market the diversity of the public health service, as well as to extend the internal vigor of the organization which originates from its diversity to the external environment. 


\section{Conclusion}

In the past decade, there has been an exponential growth in the realization that diversity management is an essential component of public management, with implications for the efficiency, competitive advantage and systematic success of the entire public service. The unique social and political history of South Africa, together with its rich cultural and ethnic diversity amplifies the challenges of managing diversity in our context. The public health service, in particular, contains a diverse corps of professions who differ on a multitude of dimensions. In addition, the intimate interface with the general population adds depth to the complexities of diversity in the public health system. The economic impetus for managing diversity is well established, but there has been a growing recognition of its potential to drive broader social change and augment the contributions of legislated programmes such as affirmative action. The challenges facing ill-prepared public managers in South Africa must be urgently addressed if we are to mount a serious and engaging response to the increasing diversity of the workforce. Sound principles of management, coupled with a commitment to diversity management at all levels are a prerequisite to any effort designed to harness the full potential of a diverse, multi-cultural human resource corps.

\section{References}

Anca, C. \& A. Vazquez 2007. Managing Diversity in the Global Organization. New York: Palgrave Macmillan.

Cavaleros, C., L. van-Vuuren \& D. Visser 2002. The Effectiveness of a

Diversity Awareness Training Programme. South African Journal of Industrial Psychology 28:50 - 61.

https://doi.org/10.4102/sajip.v28i3.68

Cilliers, F. 2007. A Systems Psychodynamic Exploration of Diversity Management: The Experiences of the Client and the Consultant. South African Journal Of Labour Relations 31:32 - 50.

Department of Health (DOH) 2006. A National Human Resource Plan for Health. Pretoria: Department Of Health (RSA).

Gay, W. \& D. Bamford 2007. A Case Study into the Management of Racial Diversity within an NHS Teaching Hospital. International Journal of Public Sector Management 20:257 - 271. 
Managing and Valuing Diversity in the SA Public Healthcare System

https://doi.org/10.1108/09513550710749996

Gill, P. 1996. Managing Workforce Diversity - A Response to Skill Shortages?

Health Manpower Management 22:34 - 37.

https://doi.org/10.1108/09552069610153107

Greybe, L. \& F. Uys 2001. Strategies for Diversity Management. Journal of Public Administration 36.

Human, L. 2005. Managing Diversity for Business Excellence. Pretoria: Van Schaik Publishers.

Hunt, B. 2007. Managing Equality and Cultural Diversity in the Health Workforce. Journal of Clinical Nursing 16:2252 - 2259.

https://doi.org/10.1111/j.1365-2702.2007.02157.x

Inyang, B. 2007. Managing Workforce Diversity and Inclusiveness in the

Public Service: Going Beyond the Nigeria Federal Character Principles (FCP). South African Journal of Labour Relations 31:85 - 101.

Maxwell, T. \& R. Rijamampianina 2002. The Sharing Principle: A Way of Managing Multicultural Organizations. South African Journal of Business Management 33:1 - 11.

Mullins, L. 2005. Management and Organisational Behaviour. Essex: Pearson Education.

Narsee, S. 2005. Durban Institute of Technology's Response to the Challenges of Multiculturalism and Diversity. South African Journal of Higher Education 182:87 - 96.

Nel, P., P.V. Dyk, G. Haasbroek, H. Schultz, T. Sono \& A. Werner 2004. Human Resources Management. Cape Town: Oxford University Press.

Penceliah, Y. 2003. Emotional Intelligence for Public Managers/ Administrators to Meet the Challenges of the Future. Public Administration: Challenges of Inequality and Exclusion. Miami: International Association of Schools And Institutes Of Administration.

Perumal, S. 1994. Affirmative Action Programmes for Business Excellence in

South Africa. Durban: Department of Business Economics, University Of

Durban-Westville.

Republic of South Africa (RSA) 2003. The National Health Act. Cape Town: Government Printer.

Schwella, E., J. Burger, W. Fox \& J. Muller 1996. Public Resource Management. Cape Town: Juta.

Siddiqui, J. \& B. Kleiner 1998. Human Resource Management in the Health Care Industry. Health Manpower Management 24:143 - 147. 
https://doi.org/10.1108/09552069810215746

Uys, I. 2003. Diversity Managment: Reasons And Challenges. Politeia 22:30 $-48$.

Walt, G. \& D. du-Toit 1997. Managing for Excellence in the Public Sector. Kenwyn: Juta.

Rubeshan Perumal Centre for the AIDS Programme of Research in South Africa Nelson R Mandela School of Medicine University of KwaZulu-Natal South Africa rubeshanperumal@gmail.com

Sadhasivan Perumal School of Management, IT and Governance University of KwaZulu-Natal South Africa Perumals@ukzn.ac.za 